\title{
PROOF OF EDREI'S SPREAD CONJECTURE
}

\author{
BY ALBERT BAERNSTEIN II
}

Communicated by W. Fuchs, November 4, 1971

A few years ago A. Edrei introduced the notion of the spread of a deficient value:

Let $f(z)$ be a meromorphic function of lower order $\mu<\infty$. (Recall that $\mu=\lim \inf _{r \rightarrow \infty}(\log T(r, f)) /(\log r)$.) Let $\left\{r_{m}\right\}$ be a sequence of Pólya peaks of order $\mu$ for $f(z)$ (see [1] for the definition of Pólya peaks), and let $\tau$ be a deficient value for $f(z), \delta(\tau, f)=\delta>0$. Put

$$
\begin{aligned}
s_{m}(\infty, f) & =\text { meas }\left\{\theta \in[-\pi, \pi]:\left|f\left(r_{m} e^{i \theta}\right)\right|>r_{m}\right\}, \\
s_{m}(\tau, f) & =s_{m}\left(\infty,(f-\tau)^{-1}\right) \quad(\tau \neq \infty) .
\end{aligned}
$$

The spread of $\tau$ is defined as

$$
\sigma(\tau, f)=\liminf _{m \rightarrow \infty} s_{m}(\tau, f) .
$$

Edrei conjectured [2, p. 57] the

Spread relation.

$$
\sigma(\tau, f) \geqq \min \left\{2 \pi, \frac{4}{\mu} \sin ^{-1} \sqrt{\frac{\delta}{2}}\right\} .
$$

He obtained an approximation $[1$, p. 83] of this inequality good enough to yield assertion I of Theorem 2 below.

The author has now obtained a proof of the exact form of the spread relation. A principal tool in this proof is the following theorem, which seems to be of independent interest.

THEOREM 1. Let $f(z)(\not \equiv 0)$ be a meromorphic function. Put

$$
m^{*}(z)=\sup _{E} \frac{1}{2 \pi} \int_{E} \log \left|f\left(r e^{i \omega}\right)\right| d \omega \quad\left(z=r e^{i \theta}, 0<\theta<\pi\right),
$$

where the sup is taken over all sets $E$ with measure exactly $2 \theta$.

Then the function

$$
T^{*}(z)=m^{*}(z)+N(|z|, f)
$$

is subharmonic in the upper half plane $\operatorname{Im} z>0$.

AMS 1970 subject classifications. Primary 30A70.

Copyright (C) American Mathematical Society 1972 
The establishment of the spread relation makes it possible for Edrei to give a solution of the "deficiency problem" for functions of small order.

THEOREM 2 (EDREI). Let $f(z)$ be a meromorphic function of lower order $\mu$, $0<\mu \leqq 1$.

I. If $0<\mu \leqq \frac{1}{2}$, then, either

$$
\sum_{\tau} \delta(\tau, f) \leqq 1-\cos \pi \mu,
$$

or else $f$ has only one deficient value (with deficiency $>1-\cos \pi \mu$ ).

II. If $\frac{1}{2}<\mu \leqq 1$, then

$$
\sum_{\tau} \delta(\tau, f) \leqq 2-\sin \pi \mu .
$$

Equality holds if and only if $f$ has only two deficient values, one of deficiency 1 , the other of deficiency $1-\sin \pi \mu$.

\section{REFERENCES}

1. A. Edrei, Sums of deficiencies of meromorphic functions, J. Analyse Math. 14 (1965), 79-107. MR 31 \#4909.

2. - Sums of deficiencies of meromorphic functions. II, J. Analyse Math. 19 (1967), 53-74. MR 35 \#6831.

Department of Mathematics, Syracuse University, Syracuse, New York 13210 